\title{
High pressure ionic and molecular crystals of ammonia monohydrate within density functional theory
}

\author{
Gareth I. G. Griffiths, Alston J. Misquitta, and Richard J. Needs \\ Theory of Condensed Matter Group, Cavendish Laboratory, \\ J J Thomson Avenue, Cambridge CB3 OHE, United Kingdom \\ A. Dominic Fortes \\ Centre for Planetary Sciences, University College London, \\ Gower Street, London WC1E 6BT, United Kingdom \\ Chris J. Pickard \\ Department of Physics and Astronomy, University College London, \\ Gower Street, London WC1E 6BT, United Kingdom
}

(Dated: November 10, 2018)

\begin{abstract}
The following article has been submitted to The Journal of Chemical Physics. After it is published, it will be found at http://jcp.aip.org/

A combination of first-principles density functional theory calculations and a search over structures predicts the stability of a proton-transfer modification of ammonia monohydrate with space group $P 4 / n m m$. The phase diagram is calculated with the PBE density functional, and the effects of a semi-empirical dispersion correction, zero point motion, and finite temperature are investigated. Comparison with MP2 and coupled cluster calculations shows that the PBE functional over-stabilizes proton transfer phases because too much electronic charge moves with the proton. This over-binding is partially corrected by using the PBE0 hybrid exchange-correlation functional, which increases the enthalpy of $\mathrm{P} 4 / \mathrm{nmm}$ by about $0.6 \mathrm{eV}$ per formula unit relative to phase I of ammonia monohydrate (AMH-I) and shifts the transition to the proton transfer phase from the PBE pressure of $2.8 \mathrm{GPa}$ to about $10 \mathrm{GPa}$. This is consistent with experiment as proton transfer phases have not been observed at pressures up to $\sim 9 \mathrm{GPa}$, while higher pressures have not yet been explored experimentally.
\end{abstract}

PACS numbers: 81.40.Vw,71.15.Nc,31.15.A-,61.66.Fn

\section{INTRODUCTION}

Ammonia monohydrate $\left(\mathrm{AMH}, \mathrm{NH}_{3} \mathrm{H}_{2} \mathrm{O}\right)$ exists as at least six different crystalline polymorphs over the experimentally studied range of pressures and temperatures of $0<p<9 \mathrm{GPa}$ and $170<T<295 \mathrm{~K} \stackrel{\underline{1}}{\text { The }}$ Thystal structures of three of these polymorphs have been determined: the low pressure phase AMH- $\mathrm{I}_{3}^{2,3}$ the high pressure disordered body-centred-cubic phase AMH-VI ${ }^{4}$ and, most recently, a combination of ab initio random structure searching (AIRSS) ${ }^{5}, \underline{6}$ and neutron powder diffraction data led to the solution of the crystal structure of AMH-II .7 .8 The structures of AMH-III, IV and V remain to be determined. The crystal structures and properties of AMH polymorphs (and of the related compound ammonia dihydrate, $\mathrm{ADH}$ ) are of interest to planetary scientists due to the likely presence of substantial fractions of ammonia in ice accreted into the satellites of the Gas Giant planets $\stackrel{9,10}{ }$ Whilst the water-rich compound $(\mathrm{ADH})$ may have a greater abundance than $\mathrm{AMH}$ at low pressures, it is known that a high-pressure form of $\mathrm{ADH}$ becomes unstable with respect to a mixture of high-pressure $\mathrm{AMH}$ and water ice at $\sim 3.5 \mathrm{GPa} \stackrel{11}{ }$ Such a pressure is relevant to the core of the large icy satellite Titan if it undifferentiated (of uniform composition) $\frac{12}{2}$ as well as during the period of its accretion $\underline{13}$ Such a pressure may also occur in the icy mantles of fully differ- entiated giant icy exoplanets or exomoons $\stackrel{14}{=} \mathrm{NH}_{3}, \mathrm{H}_{2} \mathrm{O}$ and $\mathrm{CH}_{4}$ are likely to comprise a substantial fraction of the interiors of Uranus and Neptune at pressures up to $600 \mathrm{GPa}$ and temperatures up to $7000 \mathrm{~K} \stackrel{15}{=}$ The properties of this high $p-T$ molecular mixture are thought to be important in the generation of unusual magnetic fields in these bodies $\underline{16}$

Neutron single-crystal diffraction, and the indexing and solution of a structure from powder diffraction data are non trivial at high pressure. This, combined with the tendency towards the formation and/or persistence of metastable phases in low-temperature condensed molecular systems on laboratory timescales, makes it clear that there is a central role for the computational prediction of equilibrium crystal structures and the determination of their physical properties.

In this work we combine first-principles density functional theory (DFT) calculations with a random search strategy in order to identify new candidate high-pressure AMH structures and to compute their stability with respect to one another and to other known crystalline polymorphs of AMH. Earlier DFT studies of ammonia monohydrate, ammonia hemihydrate (AHH) and solid ammonia, have revealed a propensity towards proton transfer (i.e., formation of an ionic solid) at high pressures. Calculations have suggested that AMH-I transforms to ammonium hydroxide at $\sim 5 \mathrm{GPa}, \stackrel{17}{\stackrel{\mathrm{AHH}}{\mathrm{H}} \text { transforms }}$ 
to ammonium hydroxide ammoniate at $\sim 12 \mathrm{GPa}, \frac{18}{,}$ and solid ammonia transforms to ammonium amide at $\sim 90$ GPa ${ }^{19}$ The ionic solids derived from the two hydrates are isosymmetric with their molecular precursors, but there is no reason to suppose that other ionic structures might not be energetically stable.

The presence of weak hydrogen bonds and the occurrence of both homo- and hetero-nuclear hydrogen bonds in the ammonia hydrates provide a challenge for electronic structure methods, particularly with respect to the accuracy of exchange-correlation functionals. DFT calculations using standard functionals such as the PerdewBurke-Ernzerhof (PBE) generalized gradient approximation (GGA) predict hydrogen-bonded molecular phases of AMH at low pressures, in agreement with experiment. We show here, however, that this approach predicts molecular $\mathrm{AMH}$ phases to be unstable to the formation of ionic ammonium hydroxide $\left(\mathrm{NH}_{4}^{+}: \mathrm{OH}^{-}\right)$protontransfer phases at pressures of about $2.8 \mathrm{GPa}$, although no experimental evidence for such phases has been found to date, even at pressures up to about $9 \mathrm{GPa}$. The weak van der Waals forces, which are not described by standard density functionals such as the PBE-GGA, turn out to be important in determining the volumes and relative enthalpies of the phases in this system. The zero-point (ZP) motion of the $\mathrm{H}$ atoms is also important for an accurate account of the energetics. We have, moreover, found that the most serious defect of PBE calculations in this system is that the energetics of the proton transfer is very poorly described as too much electronic charge is transferred with the proton. We show that a satisfactory description of the experimental data, including the absence of proton-transfer phases at low pressures, requires the inclusion of nuclear $\mathrm{ZP}$ motion and accurate descriptions, beyond those afforded by functionals such as PBE-GGA, of both exchange interactions and the van der Waals forces that arise from electron correlation.

\section{AB INITIO RANDOM STRUCTURE SEARCHING}

We have used the AIRSS method ${ }^{5,6}$ to identify lowenthalpy structures of AMH at pressures of up to about $12 \mathrm{GPa}$. In the AIRSS approach randomly chosen structures are relaxed to a minimum in the enthalpy at fixed pressure. In its simplest form AIRSS has almost no free parameters and is essentially unbiased, and it is therefore the ideal basis upon which to impose constraints and biases towards the types of structure that one believes are most favorable. Perhaps the simplest physical constraint that we have employed is to reject initial configurations in which atoms are closer than a defined minimum separation. One of the most useful constraints is to restrict the symmetries of the structures. The structures are chosen to obey the symmetries of a particular space group, although they are otherwise random, and the desired symmetry is maintained throughout the relaxation procedure. Another useful approach is to choose initial structures constructed from randomly placed "chemical units", which in this case are equal numbers of $\mathrm{NH}_{3}$ and $\mathrm{H}_{2} \mathrm{O}$ molecules, or equal numbers of $\mathrm{NH}_{4}$ and $\mathrm{OH}$ units, or "hydrogen-bonded" $\mathrm{NH}_{3} \cdot \mathrm{H}_{2} \mathrm{O}$ AMH units. Each initial unit cell was generated by choosing random unit cell translation vectors and scaling the volume to lie randomly within $\pm 50 \%$ of some reasonable value. We then placed the required number of chemical units within the cell, applying symmetry constraints as required. Initial structures in which the overlap of molecules was significant were rejected because they are likely to undergo unwanted chemical reactions during the relaxation procedure.

We first performed unconstrained searches with 2 formula units (f.u.), and we then searched with 4 f.u. and initial structures formed from $\mathrm{NH}_{3}$ and $\mathrm{H}_{2} \mathrm{O}$ molecules. Searches were then performed starting from random arrangements of either 2 or 4 preformed AMH units. Symmetry constrained searches were performed starting from random cells containing 2 randomly placed $\mathrm{H}_{2} \mathrm{O}$ molecules and 2 randomly placed $\mathrm{NH}_{3}$ molecules, and then applying the symmetry operations of space groups randomly chosen from those with 2 operations. Similar searches were performed with 4 symmetry operations and single units of $\mathrm{H}_{2} \mathrm{O}$ and $\mathrm{NH}_{3}$. To bias the procedure towards finding ionic structures, we started further searches with cells containing 4 f.u. and using building blocks of $\mathrm{NH}_{4}$ and $\mathrm{OH}$ units with $n=2$ or $n=4$ symmetry operations. 3 and 5 f.u. searches were performed without symmetry constraints. We also performed searches over larger unit cells containing 6, 6 and 8 f.u. in total, generated with $n=6, n=3$ and $n=8$ symmetry operations, respectively. A total of about 7,700 structures were relaxed during the searches.

The CASTEP plane wave $\operatorname{code}^{20}$ was used for all of the calculations on periodic crystals. Calculations were performed with the Perdew-Burke-Ernzerhof (PBE) generalized gradient approximation (GGA) exchangecorrelation density functional, ${ }^{21}$ the $\mathrm{PBE}$ functional with the Grimme semi-empirical dispersion correction (G06), ${ }^{22}$ and the PBE0 hybrid density functional ${ }^{23}$ which includes 25\% exact (Hartree-Fock) exchange. We used ultrasoft pseudopotentials ${ }^{24}$ for the PBE and PBE+G06 calculations and norm-conserving pseudopotentials generated using the Opium software ${ }^{25}$ for the PBE0 calculations. For the searches we used a plane wave cut off energy of $340 \mathrm{eV}$ and a Monkhorst-Pack ${ }^{26}$ Brillouin zone sampling grid of spacing $2 \pi \times 0.07 \AA^{-1}$, and $2 \pi \times 0.08$ $\AA^{-1}$ for some of the searches with larger unit cells. All of the results reported in this paper were obtained by refining the structures obtained in the searches at a higher level of accuracy consisting of a plane wave cut off energy of $700 \mathrm{eV}$ and a Brillouin zone sampling grid of spacing $2 \pi \times 0.04 \AA^{-1}$. The enthalpy difference between AMH $\mathrm{I}$ and the ionic $P 4 / \mathrm{nmm}$ phase of $\mathrm{AMH}$ reported here was changed by less than $0.0002 \mathrm{eV}$ per AMH formula unit (f.u.) on doubling the cut off energy to $1400 \mathrm{eV}$, 


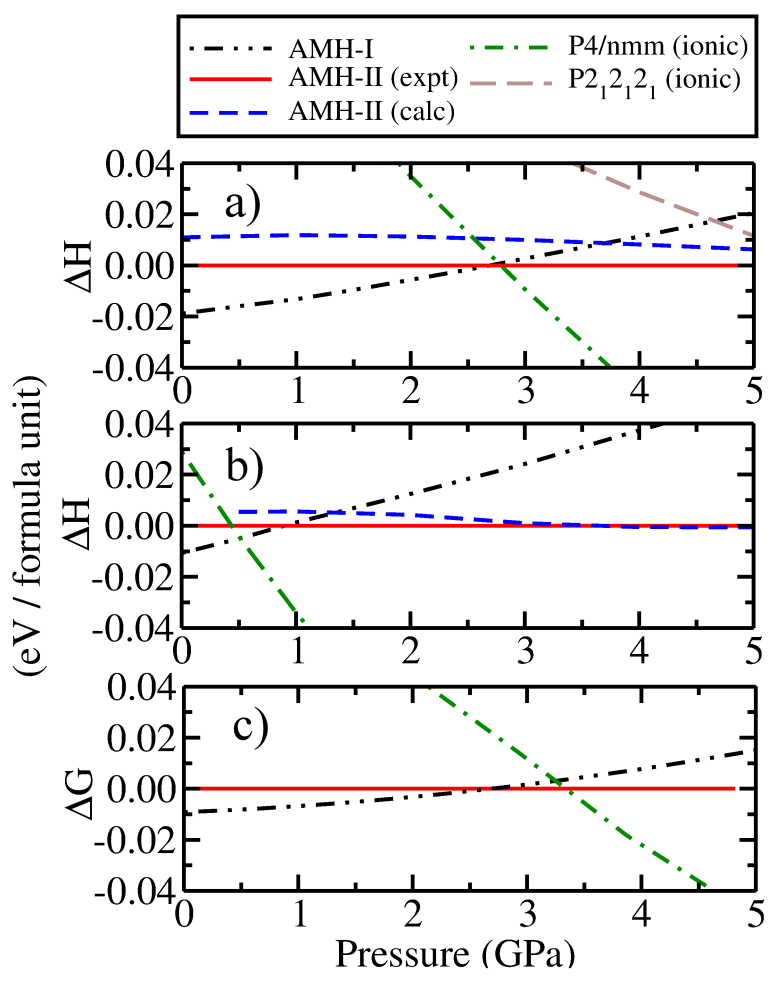

FIG. 1: Enthalpies per f.u. of AMH structures relative to AMH-II calculated with: a) the PBE functional, b) the PBE functional and G06 semi-empirical dispersion correction. The Gibbs free energy per f.u. of AMH structures relative to that of AMH-II is shown in c), calculated with the PBE functional including vibrational motion at $175 \mathrm{~K}$. AMH-II (expt) denotes the structure found in experiment while AMH-II (calc) denotes the structure found in the AIRSS study reported in Ref. $\stackrel{7}{ }$.

while the enthalpy change on doubling the number of $\mathrm{k}$-points was even smaller. The required force tolerance for a successful geometry optimization in each search run was $0.05 \mathrm{eV} / \AA$, which was tightened to $0.005 \mathrm{eV} / \AA$ for the final results reported in this paper. The stress on the unit cell was converged to better than $0.01 \mathrm{eV} / \AA^{3}$. The norm-conserving pseudopotentials required a plane wave cut off energy of $1000 \mathrm{eV}$, for which the energy difference between AMH-I and P4/nmm was converged to better than $0.00002 \mathrm{eV}$ per f.u. A less dense Brillouin zone sampling grid spacing of $2 \pi \times 0.05 \AA^{-1}$ was used for the PBE0 calculations, which gave an energy convergence of better than $0.03 \mathrm{eV}$ per f.u.

\section{RESULTS FROM STRUCTURE SEARCHING}

AIRSS was used successfully to determine the crystal structure of AMH-II in collaboration with experiment,

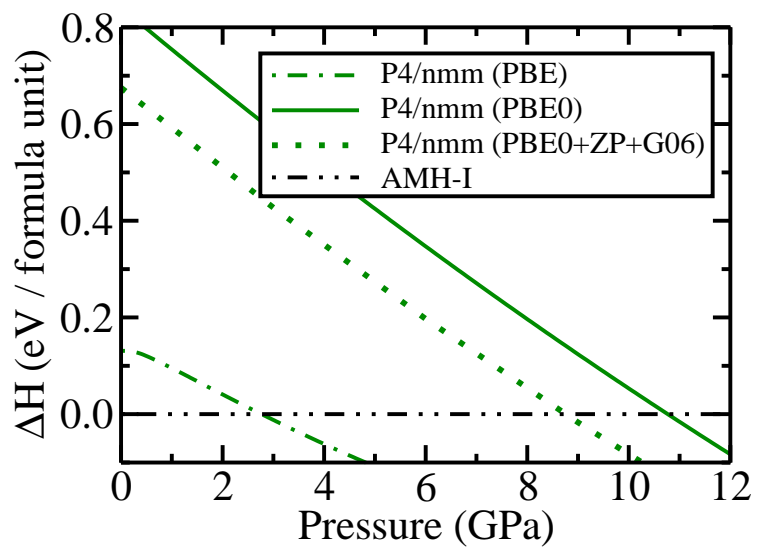

FIG. 2: Enthalpies per f.u. of $P 4 / \mathrm{nmm}$ relative to AMHI for three methods; using the PBE functional, the PBE0 hybrid functional, and PBE0 with both the G06 dispersion correction and $\mathrm{ZP}$ vibrational motion effects included. The PBE0 calculations were performed on the structures relaxed using the PBE functional.

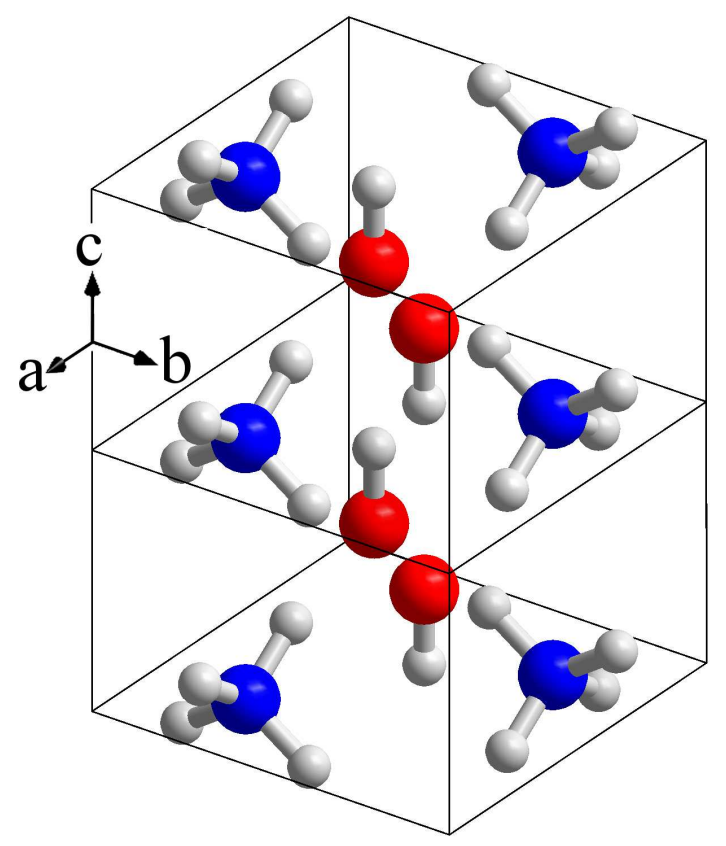

FIG. 3: The $P 4 / \mathrm{nmm}$ ionic structure; light grey atoms are hydrogen, red atoms are oxygen, and blue atoms are nitrogen. The solid lines depict two unit-cells, the orientation of the crystallographic axes being indicated on the left.

which provided initial constraints on the symmetry and dimensions of the unit cell $\stackrel{\underline{7}}{\text { The AIRSS structure with }}$ 112 atoms in the primitive unit cell was found to be almost correct in a subsequent experiment, with the exception that it produced one of the two possible $\mathrm{H}$-bond ordering schemes that is apparently not adopted by the real material. The relative enthalpies of the AMH-II structure obtained from AIRSS and the experimental structure, both fully relaxed within PBE, are shown in Fig. 1. 
The PBE and PBE+G06 calculations indicate that the AMH-II structure obtained from AIRSS is roughly 0.01 $\mathrm{eV}$ higher in enthalpy than the experimental structure. There is no reason why the experimental structure with the alternate $\mathrm{H}$-bond ordering could not have been found if more searches had been performed.

Fig. 1(a) shows the variation of the enthalpy with pressure for a number of AMH phases calculated with the PBE functional. Our searches did not find any molecular structures more stable than AMH-I and II. This may suggest that, if any of the three unsolved AMH polymorphs are molecular, they are likely to have complex architectures with $Z^{\prime}>8$, where $Z^{\prime}$ is the number of molecules in the asymmetric unit cell. The ionic ammonium hydroxide structure (space group $P 2_{1} 2_{1} 2_{1}$ ) was obtained in the DFT study of Fortes et al. ${ }^{17}$ by compressing AMH-I which underwent an isosymmetric transition to the ionic (proton-transfer) form manifested by a discontinuity in the slope of the calculated energy-volume curve. This transition was also observed in the present work. The ionic $P 2_{1} 2_{1} 2_{1}$ structure becomes more favourable than either AMH-I or AMH-II above about $6 \mathrm{GPa}$. Although the $P 2_{1} 2_{1} 2_{1}$ ionic phase has a region of stability on this phase diagram relative to the known phases, there is no reason to believe that it is the most stable ionic phase of AMH, which motivates a systematic search, as described above. Searching with AIRSS revealed a structure (shown in Fig. 3) with space group $P 4 / \mathrm{nmm}$ to be the most stable in all $2 \mathrm{f}$.u. searches at $10 \mathrm{GPa}$. Subsequent searches with $4 \mathrm{f} . \mathrm{u}$. at both 3 and $10 \mathrm{GPa}$ also showed $P 4 / \mathrm{nmm}$ to be the most stable structure, regardless of the constraints imposed. Even searches with $8 \mathrm{f}$.u. found $P 4 / \mathrm{nmm}$ to be the most stable. None of the searches performed with 3,5 , or 6 f.u. resulted in structures with enthalpies as low as that of $P 4 / \mathrm{nmm}$. In fact none of the space groups with 3 symmetry operations are subgroups of $P 4 / \mathrm{nmm}$, and therefore it could not have been found in searches with 3 symmetry operations.

The stability range of AMH-II between the AMH-I and ionic $P 4 / \mathrm{nmm}$ phases predicted by the PBE functional is very small ( $\sim 0.01 \mathrm{GPa}$, see Fig. 1). However, experiments have shown that the transition from AMH-I to AMH-II takes place at around $0.5 \mathrm{GPa}$, and then from AMH-II to AMH-IV (whose structure is presently unknown) at around $2.2 \mathrm{GPa}$ at $170 \mathrm{~K} \stackrel{1,7}{1}$ An experimental study has shown that warming AMH-IV at $6.5 \mathrm{GPa}$ produces the body-centred-cubic (bcc) phase VI at $\sim 280$ $\mathrm{K}, \frac{1}{1}$ the structure of which has been reported to consist of orientationally and positionally disordered $\mathrm{NH}_{3}$ and $\mathrm{H}_{2} \mathrm{O}$ molecules. ${ }^{4}$ Our neutron powder diffraction study has shown that compression of AMH-V at room temperature does not lead to the formation of AMH-VI up to $\sim 9 \mathrm{GPa} .27$ Interestingly, a similar disordered bcc phase of $\mathrm{ADH}$ was reported by Fortes et $a l .^{28}$ and confirmed recently by Loveday et $a l^{29}$ It is likely that a solid solution could exist between the AMH and ADH compositions at high pressures, if the bcc crystal structure is maintained over a range of occupancies of the $\mathrm{NH}_{3}$ and $\mathrm{H}_{2} \mathrm{O}$ molecules.

Clearly, construction of a complete computational phase diagram requires simulation of the AMH-VI structure. However, the disordered nature of AMH-VI precludes straightforward investigation using DFT but, as a first approximation, a so-called "shaking" search was performed, in which a $2 \times 2 \times 2$ supercell comprising only the oxygen and nitrogen atoms was created. For each search the appropriate number of hydrogen atoms were distributed randomly over the supercell and the atomic positions were relaxed. The lowest enthalpy structure, which was obtained repeatedly, does not appear in Fig. 1 as it lies approximately $0.2 \mathrm{eV}$ per f.u. above AMH-II and is quite far from thermodynamic stability. The large discrepancy between the computed stability and the reproducible experimental observation of AMH-VI is likely to be due to the small cell used in the calculations.

PBE calculations predict the ionic $P 4 / \mathrm{nmm}$ phase to be thermodynamically stable across a broad region of the high pressure phase diagram. Whether $\mathrm{P} 4 / \mathrm{nmm}$ corresponds to any of the polymorphs with as-yet undetermined structures (III, IV, or V), and its relationship (if any) to AMH-VI is not yet clear due to the lack of suitable experimental data. The published neutron powder diffraction data for AMH-III, IV, and V are not of high quality; $;, 3$ in particular the published powder pattern of AMH-IV consists only of very broad reflections. We have therefore carried out our own neutron powder diffraction study, ${ }^{27}$ with the aim of obtaining high resolution data from these polymorphs. The results acquired so far confirm that none of these polymorphs is likely to be the $P 4 / \mathrm{nmm}$ phase. There is, however, a discernible relationship between the structures of the $P 4 / \mathrm{nmm}$ phase and AMH-VI, as described below. It is worth noting that apparently small structural changes, particularly those that break a crystal symmetry, can significantly affect a diffraction pattern. Therefore it is reasonable to expect the diffraction patterns of $\mathrm{P} 4 / \mathrm{nmm}$ and AMH-VI to differ, despite the relationship between the structures.

With reference to Fig. 4, the hydrogen-bonded layers in the $P 4 / \mathrm{nmm}$ ionic phase form a network with a square motif, which defines the tetragonal unit cell (marked in black). However, an oblique cell (dashed line) is also marked that closely approximates a cube having ammonium ions at the corners and a hydroxide ion near the center. A slight shrinkage of the tetragonal a- and $\mathbf{b}$-axes $(\sim 4.4 \%$ relative to the value given in Table II) while keeping the length of the c-axis unchanged, forms a perfect cube. Furthermore, shifting the fractional $z$-coordinate of the oxygen atom from 0.6562 to 0.5 yields a heavyatom structure of space-group $\operatorname{Pr} \overline{3} m, \mathbf{a}=3.3850 \AA$, and fractional atomic coordinates $\mathrm{N}=0,0,0$, and $\mathrm{O}$ $=0.5,0.5,0.5$. Finally, mixing the occupancies of these two sites with ammonium and hydroxyl ions gives an ionic equivalent of the AMH-VI structure. In fact, the very small differences in computed Bragg intensities between the ionic and molecular forms of AMH-VI lead us to conclude that AMH-VI may well be ionic rather than 


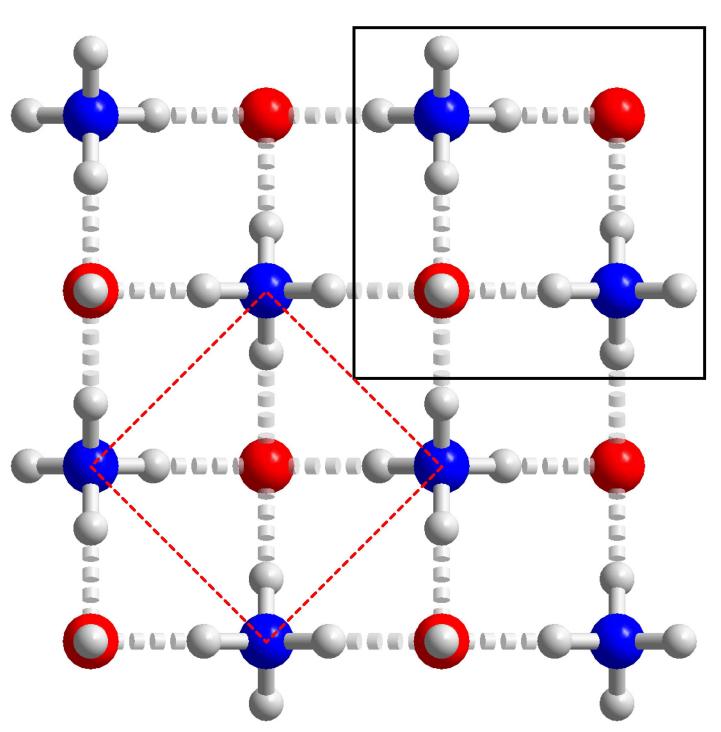

FIG. 4: The ionic $P 4 / \mathrm{nmm}$ phase viewed along the c-axis. This structure comprises sheets of ions hydrogen-bonded together (dashed rods) donated by the ammonium ions to the hydroxide. The hydrogen atom of the hydroxide ion points directly along the c-axis and does not appear to participate in a hydrogen bond. Hence, the layers are not $\mathrm{H}$-bonded to one another and consequently the structure exhibits a large compressibility along the c-axis. The dashed line shows the basis of a cubic structure that may be derived from the tetragonal cell (marked by the solid black line) by a small distortion, as discussed in the text.

molecular, and that the $P 4 / n m m$ phase might simply be an ordered variant.

\section{ZERO-POINT MOTION AND FINITE TEMPERATURE EFFECTS}

The relatively small mass of hydrogen leads to large $\mathrm{ZP}$ motions in AMH, where 5 out of every 7 nuclei are protons. The ZP motion in AMH may lead to important differences in the relative stabilities of the phases, particularly when comparing a dense ionic phase with a less dense molecular one. We have investigated the effects of ZP motion on the phase diagram of AMH within the quasi-harmonic approximation with the supercell method and finite atomic displacements. The quasi-harmonic approximation normally gives a reasonable description of vibrational effects, including thermal expansion.

We calculated the phonons of AMH-I and P4/nmm in 112 atom supercells, while a set of finite displacement phonon calculations were performed for AMH-II, which has a 112 atom primitive cell.

Care was taken to ensure that the structures were very well relaxed prior to performing phonon calculations, ensuring that any stresses on the unit cell were less than $0.01 \mathrm{eV} / \AA^{3}$ and that the forces were converged to within $0.005 \mathrm{eV} / \AA(0.003 \mathrm{eV} / \AA$ for $\mathrm{AMH}-\mathrm{II})$. In addition, the

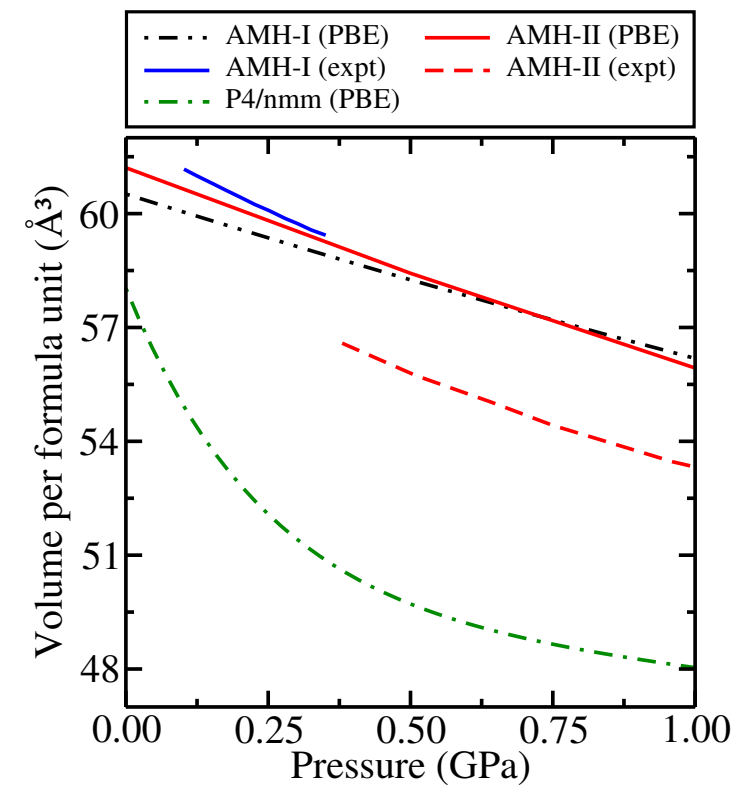

FIG. 5: Volume-pressure relationships for the AMH-I and AMH-II phases and the new ionic phase of AMH reported here, calculated with the PBE functional, including the effects of $\mathrm{ZP}$ motion and temperature $(175 \mathrm{~K})$. The experimental data were obtained from deuterated samples at $180 \mathrm{~K}$ at the Institut Laue Langevin, in the gas phase with a highresolution powder diffractometer, and from a Paris-Edinburgh cell study with a high-intensity powder diffractometer, for AMH-I and AMH-II, respectively.

fine grid on which the augmentation charge density for the ultrasoft pseudopotentials is representated was increased to 2.75 times the multiple of the wavefunction grid to obtain higher accuracy.

The Gibbs free energy at $175 \mathrm{~K}$ is plotted against pressure for AMH-I, AMH-II and the P4/nmm ionic phase in Fig. 1, as calculated with the PBE functional. The phonon pressure was evaluated from the derivative of the ZP energy (or Helmholtz free energy at finite temperature) with respect to volume. The total pressure is the sum of the static DFT and phonon pressures. The larger density of the ionic $P 4 / \mathrm{nmm}$ phase leads to higher phonon frequencies and hence destabilization relative to the molecular phases. The pressure obtained within PBE at which $P 4 / \mathrm{nmm}$ becomes the most stable at $175 \mathrm{~K}$ is increased by $0.55 \mathrm{GPa}$ to $3.32 \mathrm{GPa}$. The inclusion of vibrational effects increases the pressure interval of stability of AMH-II to about $0.7 \mathrm{GPa}$, although this is still smaller than the experimental interval of about 1.7 $\mathrm{GPa}, \underline{1,7}$

Experimentally, the transition from AMH-I to AMH-II at $\sim 0.35 \mathrm{GPa}$ results in a volume decrease of $4.6 \% . \frac{8}{\text { Both }}$ $\mathrm{PBE}$, and PBE with ZP motion at $175 \mathrm{~K}$, give only a $2 \%$ decrease in volume at this transition, whereas PBE with the G06 dispersion correction gives a decrease of 3.4\%.

Data from fits of the calculated pressure-volume data to the third-order Birch-Murnaghan equation of state, $\stackrel{30}{=}$ 


\begin{tabular}{|c|c|c|c|c|}
\hline Structure & Method & $K(\mathrm{GPa})$ & $K^{\prime}$ & $V_{0}\left(\AA^{3}\right)$ \\
\hline \multirow[t]{6}{*}{ AMH-I $(Z=4)$} & PBE & 9.7 & 5.0 & 245.04 \\
\hline & $\mathrm{PBE}+\mathrm{ZP}(0 \mathrm{~K})$ & 12.5 & 2.4 & 241.50 \\
\hline & $\mathrm{PBE}+\mathrm{ZP}(175 \mathrm{~K})$ & 12.2 & 2.4 & 242.17 \\
\hline & $\mathrm{PBE}+\mathrm{G} 06$ & 12.5 & 6.3 & 218.10 \\
\hline & Experiment $(140 \mathrm{~K})^{\frac{1}{1}}$ & $8.9(4)$ & $4.2(3)$ & 247.66 \\
\hline & Experiment $(180 \mathrm{~K})^{7}$ & $7.33(3)$ & 5.3 & $248.00(2)$ \\
\hline \multirow[t]{5}{*}{ AMH-II $(Z=16)$} & PBE & 8.1 & 5.5 & 971.60 \\
\hline & $\mathrm{PBE}+\mathrm{ZP}(0 \mathrm{~K})$ & 9.6 & 3.1 & 973.22 \\
\hline & $\mathrm{PBE}+\mathrm{ZP}(175 \mathrm{~K})$ & 9.2 & 3.0 & 983.25 \\
\hline & $\mathrm{PBE}+\mathrm{G} 06$ & 13.4 & 4.2 & 842.70 \\
\hline & Experiment $(180 \mathrm{~K})$ & $7.2(3)$ & $5.3(2)$ & $947(2)$ \\
\hline \multirow[t]{4}{*}{$P 4 / n m m(Z=2)$} & $\mathrm{PBE}$ & 3.8 & 13.8 & 106.51 \\
\hline & $\mathrm{PBE}+\mathrm{ZP}(0 \mathrm{~K})$ & 4.6 & 7.4 & 113.13 \\
\hline & $\mathrm{PBE}+\mathrm{ZP}(175 \mathrm{~K})$ & 4.5 & 5.4 & 117.95 \\
\hline & $\mathrm{PBE}+\mathrm{G} 06$ & 20.8 & 11.0 & 83.01 \\
\hline
\end{tabular}

TABLE I: The bulk modulus $(K)$, the first pressure derivative of the bulk modulus $\left(K^{\prime}\right)$, and the equilibrium volume $\left(V_{0}\right)$. Fitting ranges: AMH-I 0-2.5 GPa, AMH-II 0.4-2.5 GPa. P4/nmm PBE 3-6 GPa, PBE+G06 0-2.5 GPa.

\begin{tabular}{|c|c|c|c|c|c|c|c|c|c|}
\hline \multirow{2}{*}{$\begin{array}{c}\text { Functional } \\
\text { PBE }\end{array}$} & \multicolumn{3}{|c|}{$\begin{array}{c}\text { Lattice parameters } \\
\left(\AA \stackrel{\circ}{\circ}^{\circ}\right)\end{array}$} & \multirow{2}{*}{$\begin{array}{l}\text { Atom } \\
\mathrm{N}\end{array}$} & \multirow{2}{*}{$\begin{array}{l}\text { Site } \\
-42\end{array}$} & \multirow{2}{*}{$\begin{array}{l}\text { Wyckoff } \\
\text { symbol } \\
2 \mathrm{a}\end{array}$} & \multicolumn{3}{|c|}{ Fractional atomic coordinates } \\
\hline & $a=5.006$ & $b=5.006$ & $c=3.385$ & & & & 0.25 & 0.7500 & 0.0000 \\
\hline & & & & $\mathrm{H} 1$ & $4 m m$ & $2 \mathrm{c}$ & 0.75 & 0.7500 & 0.6308 \\
\hline & & & & $\mathrm{H} 2$ & $m$ & $8 \mathrm{i}$ & 0.25 & 0.5727 & 0.8262 \\
\hline
\end{tabular}

TABLE II: Structure of the $P 4 / \mathrm{nmm}$ ionic phase at $3 \mathrm{GPa}$ with the PBE functional (primitive cell, $Z=2$ ).

with and without the ZP motion and temperature contributions, are shown in Table I. The uncorrected PBE results compare most favorably with the experimental equilibrium volume, bulk modulus and the first pressure derivative of the bulk modulus for both AMH-I and AMH-II. It is interesting to note that there is one exception to the expected increase in volume from including ZP motion, AMH-I is seen to shrink slightly when ZP motion is taken into account. All three structures are found to increase in volume when thermal effects are included at $175 \mathrm{~K}$, accompanied by a small reduction in the bulk moduli relative to the values obtained on including $\mathrm{ZP}$ motion at $0 \mathrm{~K}$.

\section{DISPERSION CORRECTION}

The transition pressure between the AMH-I and II molecular phases obtained with PBE of $2.8 \mathrm{GPa}$ is significantly larger than the experimental value of $0.5 \mathrm{GPa}$. Here we explore the effects of including dispersion forces

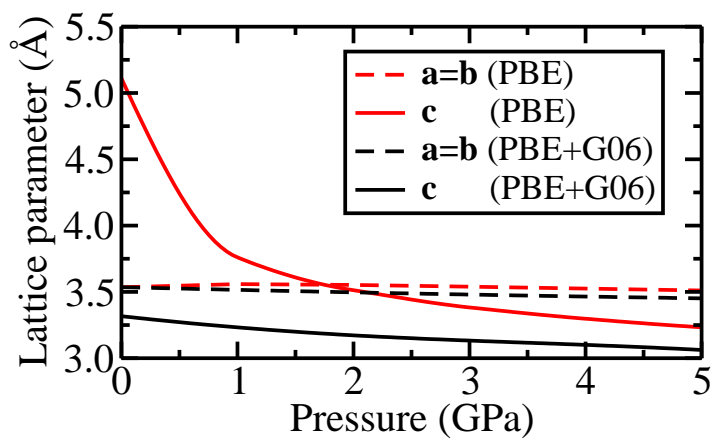

FIG. 6: Variation of the lattice parameters with pressure for the $P 4 / \mathrm{nmm}$ ionic phase, calculated with the PBE functional, with and without the G06 dispersion correction.

which are not described by density functionals such as PBE. For this purpose we have recalculated the phase diagram using the PBE functional with the Grimme semiempirical dispersion correction (G06) $\stackrel{22}{\rightleftharpoons}$ see Fig. 1 b). The significant overestimate of the transition pressure 


\begin{tabular}{l|ccc}
\hline \hline Method & & Transition Pressure $(\mathrm{GPa})$ & \\
& $\mathrm{AMH} \mathrm{I} \rightarrow \mathrm{AMH} \mathrm{II}$ & AMH II $\rightarrow P 4 / n m m$ & $2.8^{\dagger}$ \\
\hline PBE & 2.7 & 2.8 & 0.5 \\
PBE+G06 & $0.9^{\dagger}$ & $0.4^{\dagger}$ & $3.3^{\dagger}$ \\
PBE+ZP $(175 \mathrm{~K})$ & 2.7 & 3.3 & 10.8 \\
PBE0 & $\mathrm{n} / \mathrm{a}$ & $\mathrm{n} / \mathrm{a}$ & 8.8 \\
PBE0+ZP+G06 $(0 \mathrm{~K})$ & $\mathrm{n} / \mathrm{a}$ & $\mathrm{n} / \mathrm{a}$ & $\mathrm{n} / \mathrm{a}$ \\
Experiment $(180 \mathrm{~K})^{\underline{7}}$ & 0.5 & $\mathrm{n} / \mathrm{a}$ & \\
\hline \hline
\end{tabular}

TABLE III: Summary of the transition pressures between the AMH phases studied here, with each of the methods utilised. Transitions which would occur at a pressure where neither phase is thermodynamically stable are denoted by ${ }^{\dagger}$. Note that PBE0 calculations were not performed for the AMH II phase and are therefore not included in the table (n/a).

obtained with PBE is substantially improved by including the G06 correction. The transition pressure between the molecular AMH-I and AMH-II phases is reduced to 1 GPa using the PBE+G06 functional, which is still somewhat larger than the experimental value of $0.5 \mathrm{GPa}$. However, the G06 correction significantly favours the denser $P 4 / \mathrm{nmm}$ ionic phase, which now becomes stable at about $0.5 \mathrm{GPa}$. Each phase undergoes a substantial volume contraction when the G06 correction is included, which is likely due to an overestimation of dispersion effects.

The large effect of the dispersion correction on the ionic structure may seem paradoxical as it amounts to a relatively small fraction of the binding energy of the $\mathrm{NH}_{4}^{+} \ldots \mathrm{OH}^{-}$ionic complex (see Fig. 8). The rapid reduction in the volume of $P 4 / \mathrm{nmm}$ with applied pressure apparent in Fig. 5 is almost entirely associated with a contraction along the c lattice parameter. As shown in Fig. 4, the hydrogen bonds form a square net within the a-b-planes of $P 4 / \mathrm{nmm}$, but there is no apparent hydrogen bonding between the layers (see Fig. 33), and therefore it is very soft in the c direction.

\section{BEYOND THE PBE FUNCTIONAL}

\section{A. Calculations for molecular and ionic fragments}

Despite giving a good description of the bulk structural properties of AMH-I and II, the PBE functional leads to substantially incorrect transition pressures. PBE overestimates the pressure of the AMH-I/II transition, and the $\mathrm{P} 4 / \mathrm{nmm}$ ionic phase is predicted to be sufficiently stable to virtually eliminate the region of stability of AMH-II. Even accounting for ZP motion and thermal effects it is hard to reconcile the apparent stability of the $P 4 / \mathrm{nmm}$ ionic phase with the existing room temperature experiments in which the ionic AMH-VI phase was not found at pressures as high as $9 \mathrm{GPa} .31$ The structures of AMHIII, IV and V have not yet been solved, and one or more of these phases may be ionic.

To obtain insight into this problem we have selected fragments of the AMH-I and $P 4 / \mathrm{nmm}$ crystals (at pressures of 1 and $4 \mathrm{GPa}$, respectively) for a more detailed analysis. These fragments were chosen to be representative of the interactions in the crystal and consisted of pairs of molecules/ions in close proximity: $\mathrm{NH}_{3}$ and $\mathrm{H}_{2} \mathrm{O}$ for the AHM-I crystal (Fig. 7) and $\mathrm{NH}_{4}^{+}$and $\mathrm{OH}^{-}$ions for the $P 4 / \mathrm{nmm}$ crystal (Fig. 8). In Figs. 7 and 8 we plot interaction energies for these pairs calculated using the supermolecular approach, that is, $E_{\text {int }}=E(A B)-$ $E(A)-E(B)$, where $E(A)$ and $E(B)$ are the energies of the monomers and $E(A B)$ is the energy of the complex. We have used the CCSD(T), MP2, PBE, PBE+G06 and PBE0 methods, though the MP2 results are not shown as they are essentially identical to the $\operatorname{CCSD}(\mathrm{T})$ values. We have calculated counterpoise-corrected interaction energies using the Boys and Bernardi ${ }^{32}$ scheme with a SadlejpVTZ basis 33 augmented with a small set of "bond centered functions" 34 which help to saturate the dispersion energy ${ }^{35}$ Selected calculations with the larger aug-ccpVTZ basis set suggest that the $\operatorname{CCSD}(\mathrm{T})$ interaction energies are converged to $5 \%$ at the equilibrium geometry and better at larger separations. These calculations were performed using the DALTON 2.0 program 36 For deeper insight into the nature of the interaction energies we have additionally used the CAMCASP program ${ }^{37}$ to perform symmetry adapted perturbation theory (SAPT) $\mathrm{DFT}^{38-40}$ calculations to decompose the interaction energies into physical components.

Fig. 7 shows that $\mathrm{PBE}$ overbinds the $\mathrm{NH}_{3} \cdots \mathrm{H}_{2} \mathrm{O}$ complex, although the equilibrium bond length agrees reasonably well with the CCSD $(\mathrm{T})$ value. The overestimation of the bulk modulus $(K)$ of AMH-I reported in Table 1 may be a reflection of the overbinding of $\mathrm{PBE}$ and the consequent overestimate of the curvature of the potential well. In contrast, PBE0 underbinds the complex, but results in an equilibrium separation in near perfect agreement with the CCSD(T) calculations. The SAPT(DFT) energy decomposition shows that asymptotically the $\mathrm{NH}_{3} \cdots \mathrm{H}_{2} \mathrm{O}$ interaction is dominated by the dipole-dipole electrostatic energies with the dispersion and polarization energies being negligible. Consequently it should not be a surprise that both the correlated and density-functional methods agree in this region. 


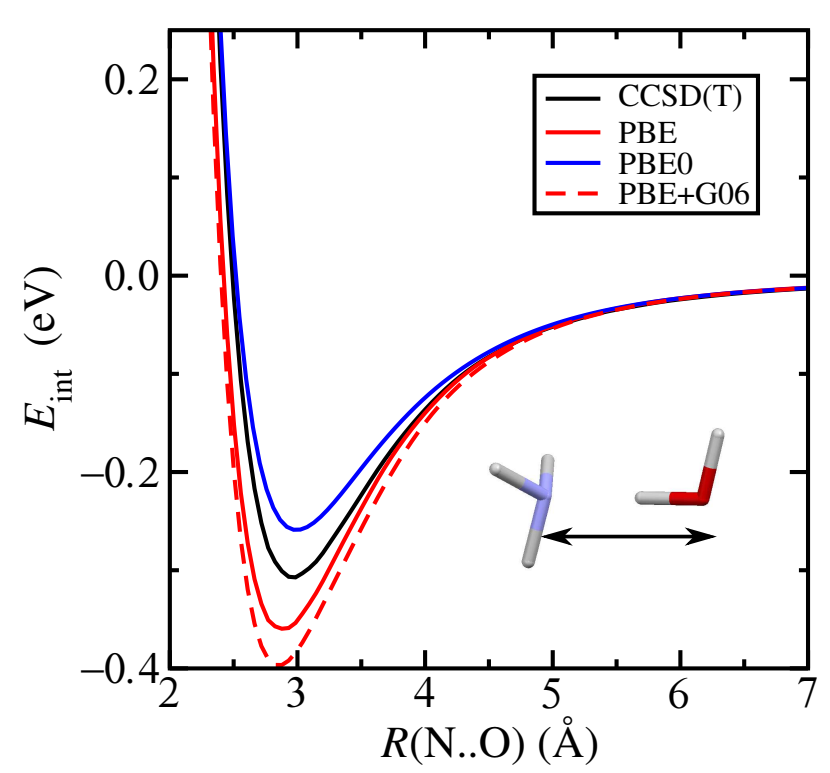

FIG. 7: Interaction energy against separation of $\mathrm{NH}_{3}$ and $\mathrm{H}_{2} \mathrm{O}$ for a configuration taken from the AMH-I molecular phase.

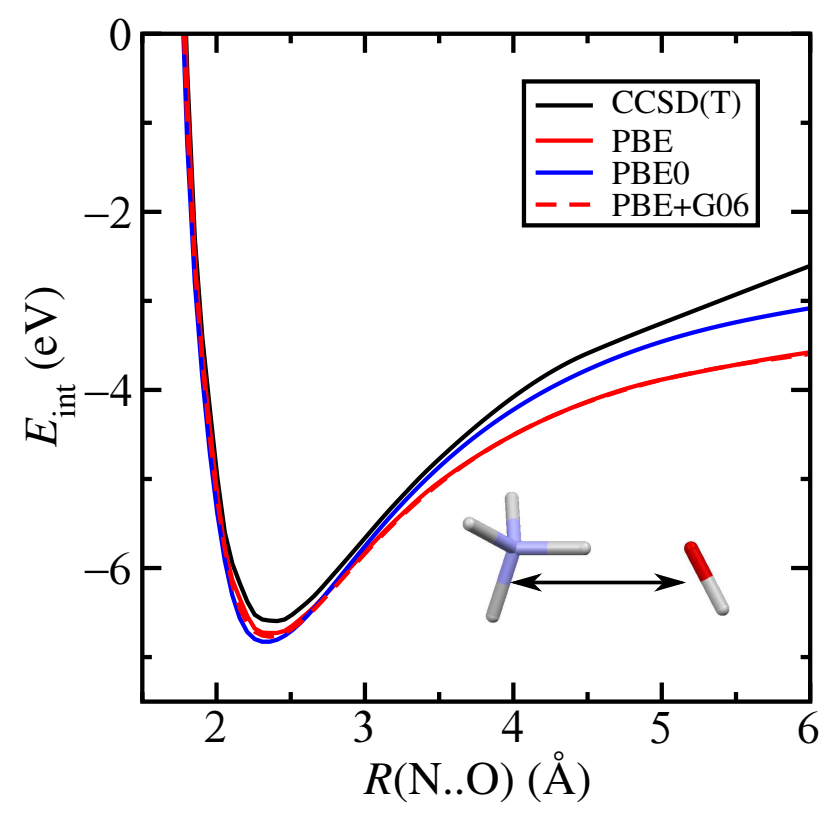

FIG. 8: Interaction energy against separation of $\mathrm{NH}_{4}^{+}$and $\mathrm{OH}^{-}$for a configuration taken from the $P 4 / \mathrm{nmm}$ ionic phase.

The ionic system behaves very differently from the molecular one. Note that the interaction energies for the ionic system shown in Fig. 8 are an order of magnitude larger than in the molecular system. The SAPT(DFT) results show that the energetics are almost completely dominated by the charge-charge electrostatic interaction between the ions, with the dispersion energy contribution being insignificant even at the relatively short $\mathrm{N} \cdots \mathrm{O}$ separation of $2.5 \AA$. This is exactly the kind of system for which local and semi-local density functionals are ex- pected to achieve high accuracy. Indeed, the PBE and PBE0 energies are close to the $\operatorname{CCSD}(\mathrm{T})$ result at the equilibrium geometry and on the repulsive wall. In contrast to the molecular system, however, PBE significantly overbinds the ionic complex at large separations. As can be seen in Fig. 8 the overbinding compared with $\operatorname{CCSD}(\mathrm{T})$ is $20 \%$ at $5 \AA$ but it grows to more than $50 \%$ at $6.5 \AA$. The interaction energies are still relatively large at these separations, and consequently these errors have important effects within the crystal. This is consistent with our observation that the $P 4 / \mathrm{nmm}$ ionic phase is over-stabilized with respect to the molecular phases.

A partial charge analysis shows that the substantial difference between the PBE and $\operatorname{CCSD}(\mathrm{T})$ interaction energies at large separations is a consequence of excessive charge transfer. Within PBE the magnitude of the charges on the monomers grows as they are pulled apart, becoming as large as $\pm 1.2 e$ at a $\mathrm{N} \cdots \mathrm{O}$ separation of 6.5 $\AA$ A. In contrast, the $\operatorname{CCSD}(\mathrm{T})$ potential energy curve can be fitted very well with a $-1 / R$ form corresponding to partial charges of $\pm 1 e$. The PBE charge transfer error is analogous to the delocalization or static correlation errors exhibited by local and semi-local density functionals $\underline{41}$ This error can be at least partially corrected by introducing some fraction of non-local exchange. Using PBE0 gives a significant improvement upon the PBE energies, with the overbinding compared with $\operatorname{CCSD}(\mathrm{T})$ at $5 \AA$ and $6.5 \AA$ reduced to $6 \%$ and $28 \%$, respectively, see Fig. 8.

\section{B. PBE0 calculations for crystalline $\mathrm{AMH}$}

As the PBE0 functional appears to offer a partial solution to the charge transfer errors which arise with $\mathrm{PBE}$, we performed calculations for the molecular AMH-I and ionic $\mathrm{P} 4 / \mathrm{nmm}$ phases using the PBE0 hybrid functional. Because of the slow convergence of the exchange terms with distance, the PBE0 calculations for the crystal were as much as two orders of magnitude more computationally expensive than the corresponding PBE calculations. For this reason geometry optimizations were not possible with PBE0 and instead we performed single-point energy calculations using the relaxed PBE structures. We attempted to obtain equation of state parameters from the PBE0 results for AMH-I and P4/nmm. The calculated energies were fitted to the third-order Birch-Murnaghan equation of state and the pressure was obtained by differentiation. We obtained equilibrium volumes of $V_{0}=220$ $\AA^{3}$ for AMH-I and $V_{0}=172 \AA^{3}$ for $P 4 / n m m$, which are similar to those obtained from the $\mathrm{PBE}+\mathrm{G} 06$ calculations. We were not able to obtain reliable estimates of $K$ or $K^{\prime}$ from our PBE0 data as the values were sensitive to the data and fitting procedure. The use of the PBE structures for the PBE0 calculations is an approximation. However, we believe this approach to be reasonably robust because enthalpies obtained using partially relaxed PBE structures were almost identical to those from the 


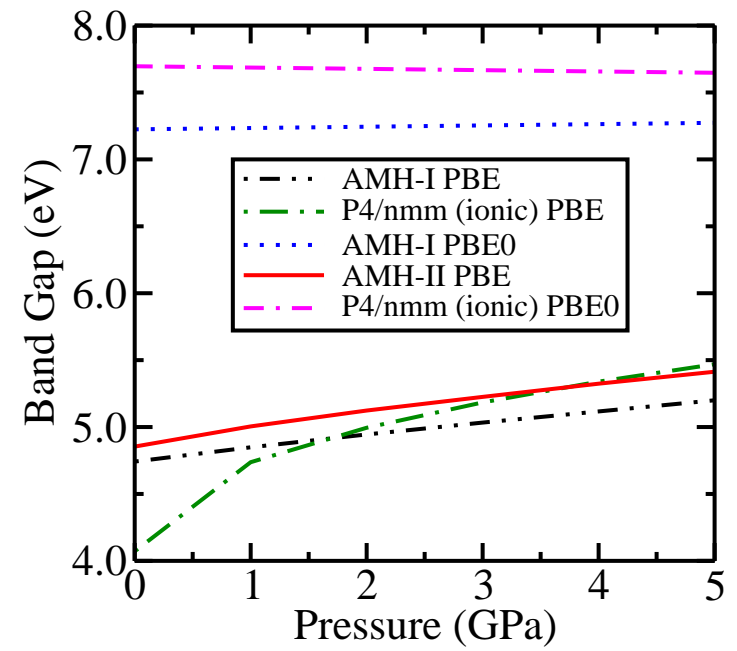

FIG. 9: Variation of the band gaps of the phases with pressure. AMH-I (and AMH-II using PBE only) have direct band gaps, while both the PBE and PBE0 calculations predict that the $P 4 / \mathrm{nmm}$ phase has an indirect band gap. The PBE0 calculations were performed using the PBE structures.

fully relaxed structures.

Mulliken charge analysis of the $P 4 / \mathrm{nmm}$ crystal suggests that the electronic charge transfer between the " $\mathrm{OH}^{-}$" and " $\mathrm{NH}_{4}^{+}$" ions has a magnitude of about $0.70 e$ when using the PBE functional and $0.55 e$ with PBE0. The improved description of the ionic phase provided by PBE0 destabilizes the $P 4 / \mathrm{nmm}$ ionic phase relative to AMH-I, and consequently $P 4 / \mathrm{nmm}$ enters above 10.8 GPa, as can be seen in Fig. 2 AMH-II, AMH-IV and AMH-VI are not present on this phase diagram, but they are expected to have regions of stability between AMH-I and $P 4 / n m m$, pushing the entry of the new ionic phase to even higher pressures. The inclusion of vibrational effects further destabilizes $P 4 / \mathrm{nmm}$ and it only becomes stable at $11.3 \mathrm{GPa}$, although conversely the inclusion of the G06 dispersion correction reduces the transition pressure to $8.8 \mathrm{GPa}$, as shown in Fig. 2]

\section{BAND GAPS OF THE PHASES}

The minimum band gaps obtained with the PBE and PBE0 functionals are shown in Fig. 9 calculated using the LINDOS code $\underline{\underline{42}}$ While the molecular structures have direct band gaps, both $\mathrm{PBE}$ and $\mathrm{PBE} 0$ calculations predict $P 4 / n m m$ to have an indirect band gap. The band gaps provided by the PBE0 functional, which includes explicit exchange interactions, are substantially larger than those predicted by PBE.

\section{CONCLUSIONS}

We have performed extensive $a b$ initio searches for new phases of AMH at pressures up to $12 \mathrm{GPa}$ using the
PBE functional. A new ionic structure of space group $P 4 / n m m$ was found to be stable above $2.8 \mathrm{GPa}$. Subsequent investigations into the effects of temperature, $\mathrm{ZP}$ motion and dispersion forces found that the latter two play a substantial role in determining the relative stabilities of the phases. The inclusion of ZP motion destabilizes the dense $P 4 / \mathrm{nmm}$ ionic phase; conversely the dispersion correction leads to a significant underestimation of the transition pressure from molecular AMH to ionic $P 4 / \mathrm{nmm}$. The dispersion forces are attractive and therefore tend to favour denser structures.

The relationship described earlier between the structures of the $P 4 / \mathrm{nmm}$ phase and AMH-VI may indicate that we have discovered an ordered ionic variant of AMH-VI. Alternatively, the $P 4 / \mathrm{nmm}$ phase may be related to one of the three experimentally observed AMH phases (III, IV, V) whose structures are unknown. As the $P 4 / \mathrm{nmm}$ phase was the only new structure found which is predicted to be thermodynamically stable within some pressure range, it is likely that if it is one of the three unknown AMH structures, then the other two will have unit cells with $Z>8$.

Accurate $\operatorname{CCSD}(\mathrm{T})$ calculations on representative complexes from the ionic and molecular AMH phases revealed that the PBE functional substantially overbinds the ionic phase. We have presented evidence that this overbinding arises from an overestimate of the electronic charge transfer accompanying the proton transfer, which can be partially remedied by using the hybrid PBE0 functional. Using the PBE0 functional leads to an increase in the enthalpy of the ionic $P 4 / \mathrm{nmm}$ phase by about $0.6 \mathrm{eV}$ per f.u. relative to AMH-I. The transition pressure from AMH-I to $P 4 / \mathrm{nmm}$ phase is substantially increased, which eliminates the inconsistency with experiment. This failure of the PBE functional, for an electrostatic-bound system for which GGA-type density functionals are typically assumed to be accurate, is likely to have implications for other systems. Further experimental work is necessary to explore the phase diagram of $\mathrm{AMH}$ at $9 \mathrm{GPa}$ and above to confirm our assignment of $P 4 / \mathrm{nmm}$ as a stable ionic phase of AMH.

\section{ACKNOWLEDGMENTS}

This work was supported by the Engineering and Physical Research Council (EPSRC) of the UK. Computational resources were provided by the Cambridge High Performance Computing Service. ADF acknowledges funding from the Science and Technology Facilities Council (STFC), UK, fellowship number PP/E006515/1. 
1 J. S. Loveday and
High Press. Res., 24, 45 (2004)

2 I. Olovsson and Acta Cryst., 12, 827 (1959).

3 J. S. Loveday and R. J. Nelmes, Sci. Tech. High Press. Proc. AIRAPT-17, 133 (2000).

4 J. S. Loveday and R. J. Nelmes, Phys. Rev. Lett., 83, 4329 (1999)

5 C. J. Pickard and R. J. Needs, J. Phys.: Condens. Matter, 23, 053201 (2011).

6 C. J. Pickard and R. J. Needs, Phys. Rev. Lett., 97, 045504 (2006).

7 A. D. Fortes, E. Suard, M. H. LeméeCailleau, C. J. Pickard, and R. J. Needs, J. Am. Chem. Soc., 131, 13508 (2009)

8 A. D. Fortes, E. Suard, M. H. Lemée-Cailleau, C. J. Pickard, and R. J. Needs, J. Chem. Phys., 131, 154503 (2009).

9 J. Kargel, Icarus, 100, 556 (1992)

10 A. D. Fortes and M. Choukroun, Space Sci. Rev., 153, 185 (2010).

11 A. D. Fortes, I. G. Wood, L. Vočadlo, K. S. Knight, W. G. Marshall, M. G. Tucker, and F. Fernandez-Alonso, J. Appl. Cryst., 42, 846 (2009)

12 A. D. Fortes, Planet. Space Sci., In Press, Corrected Proof,

13 J. Lunine and D. Stevenson, Icarus, 70, 61 (1987)

${ }^{14}$ R. Fu, R. O'Connell, and D. Sasselov, ApJ, 708, 1326 (2010)

15 W. Hubbard and J. MacFarlane, J. Geophys. Res., 85, 225 (1980).

16 C. Cavazzoni, G. Chiarotti, S. Scandolo, E. Tosatti, M. Bernasconi, and M. Parrinello, Science, 283, 44 (1999)

17 A. D. Fortes, J. P. Brodholt, I. G. Wood, L. Vočadlo, and H. D. B. Jenkins, J. Chem. Phys., 115, 7006 (2001)

18 A. D. Fortes, Ph.D. thesis, University of London (2004).

19 C. J. Pickard and R. J. Needs, Nature Mater., 7, 775 (2008)

20 S. J. Clark, M. D. Segall, C. J. Pickard, P. J. Hasnip, M. I. J. Probert, K. Refson, and M. C. Payne, Z. Kristallogr., 220, 567 (2005).

21 J. P. Perdew, K. Burke, and M. Ernzerhof, Phys. Rev. Lett., 77, 3865 (1996).

22 S. Grimme, J. Comp. Chem., 27, 1787 (2006)

23 C. Adamo and V. Barone, J. Chem. Phys., 110, 6158 (1999).

24 D. Vanderbilt, Phys. Rev. B, 41, 7892 (1990).

25 Opium: Pseudopotential Generation Project. http://opium.sourceforge.net/ (2011).
${ }^{26}$ H. J. Monkhorst and J. D. Pack, Phys. Rev. B, 13, 5188 (1976).

27 A. D. Fortes, Institut Laue Langevin experimental report, 5 (2010)

28 A. D. Fortes, I. G. Wood, M. Alfredsson, L. Vočadlo, K. S. Knight, W. G. Marshall, M. G. Tucker, and F. FernandezAlonso, High Press. Res., 27, 201 (2007)

29 J. S. Loveday, R. J. Nelmes, C. L. Bull, H. E. MaynardCasely, and M. Guthrie, High Press. Res., 29, 396 (2009)

30 F. Birch, Phys. Rev., 71, 809 (1947)

31 A. D. Fortes, G. I. G. Griffiths, R. J. Needs, C. J. Pickard, and T. Hansen, Institut Laue Langevin experimental report, XXII Congress and General Assembly International Union of Crystallography, IUCr2011, Madrid, Spain (2011).

32 S. F. Boys and F. Bernardi, Mol. Phys., 19, 553 (1970).

33 A. J. Sadlej, Theor. Chim. Acta, 79, 123 (1991).

${ }^{34}$ R. Bukowski, J. Sadlej, B. Jeziorski, P. Jankowski, K. Szalewicz, S. A. Kucharski, H. L. Williams, and B. M. Rice, J. Chem. Phys., 110, 3785 (1999).

35 H. L. Williams, E. M. Mas, K. Szalewicz, and B. Jeziorski, J. Chem. Phys., 103, 7374 (1995).

36 T. Helgaker, H. J. A. Jensen, P. Joergensen, J. Olsen, K. Ruud, H. Aagren, A. Auer, K. Bak, V. Bakken, O. Christiansen, S. Coriani, P. Dahle, E. K. Dal(20klok), T. Enevoldsen, B. Fernandez, C. Haettig, K. Hald, A. Halkier, H. Heiberg, H. Hettema, D. Jonsson, S. Kirpekar, R. Kobayashi, H. Koch, K. V. Mikkelsen, P. Norman, M. J. Packer, T. B. Pedersen, T. A. Ruden, A. Sanchez, T. Saue, S. P. A. Sauer, B. Schimmelpfennig, K. O. Sylvester-Hvid, P. R. Taylor, and O. Vahtras, "Dalton, a molecular electronic structure program, release 2.0," (2005), see http://www.kjemi.uio.no/software/dalton/dalton.html

37 A. J. Misquitta and A. J. Stone, "CAMCASP: a program for studying intermolecular interactions and for the calculation of molecular properties in distributed form," University of Cambridge (2007), http://wwwstone.ch.cam.ac.uk/programs.html\#CamCASP.

38 A. J. Misquitta and K. Szalewicz, Chem. Phys. Lett., 357, 301 (2002).

39 A. J. Misquitta, B. Jeziorski, and K. Szalewicz, Phys. Rev. Lett., 91, 33201 (2003).

40 A. J. Misquitta, R. Podeszwa, B. Jeziorski, and K. Szalewicz, J. Chem. Phys., 123, 214103 (2005).

41 A. J. Cohen, P. Mori-Sánchez, and W. Yang, Science, 321, 792 (2008).

42 A. J. Morris and C. J. Pickard, LINDOS - Version 1.3 User Manual, University College London (2011). 